\title{
Submarine groundwater discharge to Great South Bay, NY, estimated using $\mathrm{Ra}$ isotopes
}

\author{
Aaron J. Beck, John P. Rapaglia, J. Kirk Cochran, \\ Henry J. Bokuniewicz, and Suhui Yang \\ Marine Sciences Research Center, Stony Brook University
}

There is increasing evidence that submarine groundwater discharge (SGD) represents a major source of dissolved chemical constituents to the coastal ocean. In Great South Bay, NY, previous studies have shown that the discharge of nutrients with SGD may cause harmful algal blooms. This study estimates SGD to Great South Bay during August 2006 by performing a mass balance for each of the dissolved Ra isotopes (224Ra, 223Ra, 228Ra, 226Ra). The budget indicates a major source (between 30 and 60 percent of the steady-state flux) of Ra to the bay. This imbalance can be resolved by the input of Ra-enriched groundwater on the order of $3.1-8.1 \times 10^{\wedge} 9 \mathrm{~L} \mathrm{~d}-1$, depending on the Ra isotope. As much as $40 \%$ of this discharge is apparently due to flow along preferential flowpaths which are not included in models previously developed for SGD in Great South Bay. Compared to previous reports for fresh groundwater discharge to the bay, the Ra-estimated discharge must comprise approximately $90 \%$ recirculated seawater. The substantial source of Ra to the bay suggests that SGD may be a major pathway for input of other dissolved constituents such as trace metals, although the existence of channelized or preferential flow may make quantifying these chemical fluxes difficult. 